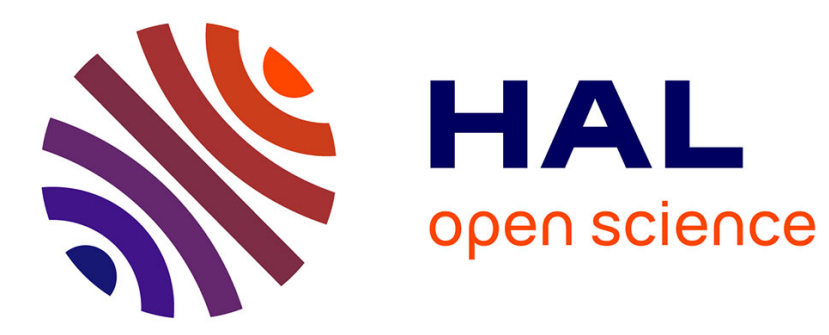

\title{
Adaptive Discontinuous Galerkin Method for Richards Equation
}

Jean-Baptiste Clément, Frederic Golay, Mehmet Ersoy, Damien Sous

\section{To cite this version:}

Jean-Baptiste Clément, Frederic Golay, Mehmet Ersoy, Damien Sous. Adaptive Discontinuous Galerkin Method for Richards Equation. Topical Problems of Fluid Mechanics 2020, Feb 2020, Prague, Czech Republic. pp.27-34, 10.14311/TPFM.2020.004 . hal-02507326

\section{HAL Id: hal-02507326 \\ https://hal.science/hal-02507326}

Submitted on 14 Mar 2020

HAL is a multi-disciplinary open access archive for the deposit and dissemination of scientific research documents, whether they are published or not. The documents may come from teaching and research institutions in France or abroad, or from public or private research centers.
L'archive ouverte pluridisciplinaire HAL, est destinée au dépôt et à la diffusion de documents scientifiques de niveau recherche, publiés ou non, émanant des établissements d'enseignement et de recherche français ou étrangers, des laboratoires publics ou privés. 


\title{
ADAPTIVE DISCONTINUOUS GALERKIN METHOD FOR RICHARDS EQUA- TION
}

\author{
J.-B. Clément ${ }^{1,2}$, F. Golay ${ }^{1}$, M. Ersoy ${ }^{1}$, D. Sous ${ }^{2,3}$ \\ ${ }^{1}$ IMATH, EA 2134, Université de Toulon, CS 60584, 83041 Toulon Cedex 9, France \\ 2 Université de Toulon, Aix Marseille Université, CNRS, IRD, Mediterranean Institute of \\ Oceanography (MIO), La Garde, France \\ ${ }^{3}$ Univ. Pau \& Pays Adour / E2S UPPA, Laboratoire des Sciences de I'Ingénieur Appliquées \\ à la Mécanique et au Génie Electrique (SIAME) - MIRA, EA4581, 64600, Anglet, France
}

\begin{abstract}
This paper deals with the numerical simulation of time-dependant flows in partially saturated porous media modelled by Richards equation. A Discontinuous Galerkin method together with an implicit scheme are used to approximate the solution. The mathematical framework and a procedure for solving this nonlinear equation are presented. In particular, treatment of seepage boundary condition and adaptive time step are underlined. An adaptive mesh refinement technique is also outlined in order to capture wetting front efficiently. Some numerical simulations are performed to illustrate the performance of the method.
\end{abstract}

Keywords: Richards equation, Discontinuous Galerkin method, seepage boundary condition, adaptive time step, adaptive mesh refinement.

\section{Introduction}

Many problems involve numerical simulation of flows in variably saturated porous media. They range from oil industry and geotechnical engineering to agriculture and earth science. Lots of models are used to study groundwater dynamics in such systems, but Richards equation-based models are widespread for saturated/unsaturated flows. As a consequence, subsurface hydrogeology community has good numerical experience about solution of Richards equation [1, 2]. The present work is the continuation of a first paper [3] and enters into a long-term research project aiming to provide insight into the groundwater circulation due to wave action on sandy beaches.

Richards equation holds strong non-linearities due to boundary conditions and constitutive laws. The latter can exhibit steep gradients or almost-zero values. Moreover, Richards equation is a parabolic equation which degenerates into an elliptic equation under complete saturation. Sharp wetting fronts can also happen according to initial and boundary conditions. Typically, designing a robust and general numerical tool can be troublesome for the solution of Richards equation [1]. First attempts to solve Richards equation go back to the early 1970s with Rubin [4] or Cooley [5] for example. Then, from 1980s, Richards equation was studied extensively, both from theoretical and numerical point of view. Results of existence, regularity and uniqueness were found [6] and solution was assessed through simulations involving different numerical methods. One of the points of interest was nonlinear solver [7, 8]. Since 2000s, works focus mainly on Richards equation stability and development of coupled and general simulations [9]. In recent years, research consists in Richards equation with hysteresis and advanced spatial/temporal discretizations $[10,11]$ with adaptive approximations in size and/or in order.

A discontinuous Galerkin method is chosen in this work. It is based on a variational formulation in an element-wise fashion. So, it is locally conservative which is crucial in fluid dynamics [12]. Moreover, it enables to change the degree of polynomial approximation and to use non-conforming mesh (hanging node). This is an important benefit since it is possible to handle high-order accuracy and $h p$-adaptation [13].

The first part of the paper introduces the Richards equation and constitutive laws along with the seepage boundary condition. Then, Richards equation is discretized with a Discontinuous Galerkin method together with a backward Euler scheme. The linearization and adaptive techniques for time and mesh are also outlined. The last part of the paper is devoted to the validation of the numerical methods, through infiltration and water table depletion simulations. 


\section{Problem formulation}

\subsection{Richards equation and constitutive laws}

Richards equation is a classic nonlinear parabolic equation to describe flow occurring in the unsaturated zone of an aquifer [1, 14]:

$$
\partial_{t}(\theta(\psi))-\nabla \cdot(\mathbb{K}(\psi) \nabla(\psi+z))=0
$$

where $\psi$ denotes the pressure head $(m), \mathbb{K}$ the hydraulic conductivity tensor $(\mathrm{m} / \mathrm{s}), z$ the elevation $(m)$ and $\theta$ the water content. Equation (1) is called the mixed form of Richards equation because it involves both variables, $\theta$ and $\psi$. This form was chosen because it is mass conservative and it is applicable to a wide range of porous media [1]. To close this system of equations, two constitutive laws are needed for water content and hydraulic conductivity. To describe these relations, it exists several models which depends on hydraulic properties of the porous medium. In this paper, the model used for the simulations $[15,16]$ is:

$$
(\mathbb{K}(\psi) ; \theta(\psi))= \begin{cases}\left(\frac{A K_{s}}{A+|\psi|^{B}} ; \frac{C\left(\theta_{s}-\theta_{r}\right)}{C+|\psi|^{D}}+\theta_{r}\right), & \text { if } \psi \leq 0 \text { (unsaturated zone), } \\ \left(K_{s} ; \theta_{s}\right), & \text { if } \psi>0 \text { (saturated zone), }\end{cases}
$$

where $A, B, C$ and $D$ are dimensionless empirical parameters, $K_{s}(\mathrm{~m} / \mathrm{s})$ is the hydraulic conductivity at saturation, $\theta_{s}$ is the saturated water content and $\theta_{r}$ is the residual water content.

Throughout this paper, hydraulic head $h(m)$ and flux $q(m / s)$ are also used: $h=\psi+z$ and $\boldsymbol{q}=-\mathbb{K}(\psi) \nabla h$. Equation (1) is rewritten as:

$$
\partial_{t}(\theta(h-z))-\nabla \cdot(\mathbb{K}(h-z) \nabla h)=0 .
$$

\subsection{Seepage boundary condition}

The seepage boundary condition is specific to subsurface model [17]. It models the interface between porous medium and atmosphere. If the porous medium is saturated and outflow occurs, then water pours out at atmospheric pressure $(\psi=0)$. Otherwise, the interface acts as an impervious boundary. The difficulty is that the length of seepage face is unknown a priori. It can be seen as a non-linear Robin boundary condition:

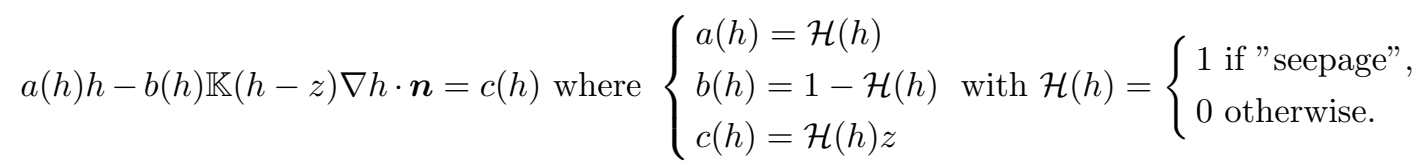

Sometimes, the Heaviside function $\mathcal{H}$ is regularized [18] to overcome convergence difficulties. The seepage boundary condition is also taken into account as a Signorini's type:

$$
\psi \leq 0, \quad-\mathbb{K}(h-z) \nabla h \cdot \boldsymbol{n} \geq 0, \quad(-\mathbb{K}(h-z) \nabla h \cdot \boldsymbol{n}) \psi=0 .
$$

In this work, the seepage boundary condition is not regularized and is treated like in [17] by switching between Dirichlet and Neumann boundary condition for each degree of freedom within the weak formulation of the next section. Therefore, where the seepage boundary condition applies, it is written:

$$
\begin{cases}h=z & \text { if } \psi \geq 0 \text { and }-\mathbb{K}(h-z) \nabla h \cdot \boldsymbol{n}>0 \\ -\mathbb{K}(h-z) \nabla h \cdot \boldsymbol{n}=0 & \text { otherwise. }\end{cases}
$$

Paniconi and Putti [8] reported that seepage condition can cause oscillations leading to convergence problems if rapid changes of saturation state occur near the seepage face. This holds no matter convergence requirements are relaxed or not for the nonlinear solver. Therefore, seepage boundary condition resolution is fully integrated into the nonlinear iterations for this study. 


\section{Discontinuous Galerkin method}

\subsection{Discretization}

First of all, some definitions are set for the discontinuous Galerkin formulation. More careful developments can be found in Rivière [12] or Dolejší and Feistauer [13]. Let $\Omega$ be a space of $\mathbb{R}^{2}$ with $\partial \Omega=\Gamma_{D} \cup \Gamma_{N}$ if no seepage boundary condition is considered. From equation (3) and by adding initial and boundary conditions, the model problem is:

$$
\left\{\begin{aligned}
\partial_{t} \theta(h-z)-\nabla \cdot(\mathbb{K}(h-z) \nabla h) & =0, & & \text { in } \Omega \times[0, T], \\
h & =h_{D}, & & \text { on } \Gamma_{D} \times[0, T], \\
-\mathbb{K}(h-z) \nabla h \cdot \boldsymbol{n} & =q_{N}, & & \text { on } \Gamma_{N} \times[0, T], \\
h & =h_{0}, & & \text { in } \Omega \times\{0\},
\end{aligned}\right.
$$

with $T$ the total duration of the simulation and $h_{0}$ the initial condition. $\Omega$ is subdivided into elements $E$ which form the partition denoted by $\mathcal{E}_{h}$. Elements are made of faces $F . \mathcal{F}_{h}^{I}$ stands for the set of interior faces, $\mathcal{F}_{h}^{D}$ for the set of Dirichlet boundary faces and $\mathcal{F}_{h}^{N}$ for the set of Neumann boundary faces.

Let be two neighbouring elements $E_{l}$ and $E_{r}$ sharing one face $F$. There are two traces of a function $u$ along $F$ denoted by $u_{l}$ and $u_{r}$. The jump across a face is denoted by $\llbracket u \rrbracket=u_{l}-u_{r}$, the mean by $\{u\}=\frac{1}{2}\left(u_{l}+u_{r}\right)$ and $\boldsymbol{n}$ is the unit normal vector oriented from $E_{l}$ to $E_{r}$.

Several discontinuous Galerkin methods are possible, see [12, 13]. Here, the incomplete interior penalty Galerkin (IIPG) method is chosen to avoid the addition of stabilization terms from symmetrization [13]. Penalization terms are added to enforce Dirichlet boundary condition and continuity constraint through respectively, the boundary penalty $\sigma_{b}$ and the interface penalty $\sigma_{i}$. Let $H^{s}\left(\mathcal{E}_{h}\right)=\left\{v \in L^{2}(\Omega): \forall E \in \mathcal{E}_{h},\left.v\right|_{E} \in H^{s}(E)\right\}$ be the broken Sobolev space with $H^{s}$ the classic Sobolev space and $s>3 / 2$. Problem (7) is multiplied by a test function $v \in H^{s}\left(\mathcal{E}_{h}\right)$, integrated on each element $E$. After using Green's theorem, summing over all elements and performing some calculations, the space semi-discretization is obtained:

$$
\begin{aligned}
& \text { Find } h \in H^{s}\left(\mathcal{E}_{h}\right) \text { such that } \forall v \in H^{s}\left(\mathcal{E}_{h}\right), \quad \sum_{E \in \mathcal{E}_{h}} \int_{E} \partial_{t} \theta(h-z) v \mathrm{~d} \boldsymbol{x}+a_{h}(h, v)=l_{h}(v) \text { with } \\
& a_{h}(h, v)= \sum_{E \in \mathcal{E}_{h}} \int_{E} \mathbb{K}(h-z) \nabla h \cdot \nabla v \mathrm{~d} \boldsymbol{x}-\sum_{F \in \mathcal{F}_{h}^{I}} \int_{F}\left\{\mathbb{K}(h-z) \nabla h \cdot \boldsymbol{n}_{\boldsymbol{F}} \rrbracket \llbracket v \rrbracket \mathrm{d} s+\sum_{F \in \mathcal{F}_{h}^{I}} \int_{F} \frac{\sigma_{i}^{F}}{|F|} \llbracket h \rrbracket \llbracket v \rrbracket \mathrm{d} s\right. \\
&-\sum_{F \in \mathcal{F}_{h}^{D}} \int_{F} \mathbb{K}(h-z)\left(\nabla h \cdot \boldsymbol{n}_{\boldsymbol{F}}\right) v \mathrm{~d} s+\sum_{F \in \mathcal{F}_{h}^{D}} \int_{F} \frac{\sigma_{b}^{F}}{|F|} h v \mathrm{~d} s \\
& l_{h}(v)= \sum_{F \in \mathcal{F}_{h}^{D}} \int_{F} \frac{\sigma_{b}^{F}}{|F|} h_{D} v \mathrm{~d} s-\sum_{F \in \mathcal{F}_{h}^{N}} \int_{F} q_{N} v \mathrm{~d} s
\end{aligned}
$$

Contrary to the Finite Elements method, there is no continuity constraint between elements for the test functions. Therefore, any polynomial basis functions is suitable which makes discontinuous Galerkin method flexible. Even if a nodal basis can be chosen, this paper works with a modal basis, the monomial basis, because it is simple and can be extended easily to higher orders.

To get the full discretization, the first term of the weak formulation (8) is approximated by a backward Euler scheme which gives:

Find $h \in H^{s}\left(\mathcal{E}_{h}\right)$ such that $\forall v \in H^{s}\left(\mathcal{E}_{h}\right)$,

$$
R_{h}\left(h^{n+1}, v\right)=\sum_{E \in \mathcal{E}_{h}} \int_{E} \frac{\theta\left(h^{n+1}-z\right)-\theta\left(h^{n}-z\right)}{\delta t} v \mathrm{~d} \boldsymbol{x}+a_{h}\left(h^{n+1}, v\right)-l_{h}\left(v ; t^{n+1}\right)=0,
$$

where $\delta t$ is the time step, $n$ is the number of time iteration and $h^{n}$ denotes $h$ at time $t_{n}$. The choice for backward Euler scheme is quite natural because its implicit nature provides natural stability properties. Together with its simple implementation, this scheme is widespread for Richards equation where good convergence behaviour is observed in [8]. 


\subsection{Nonlinear solver with adaptive time stepping}

Solving equation (11), which represents the residual $R$, can be challenging because of strong nonlinearities. Newton-Raphson methods converge quadratically but they often fail for parabolic degenerate problem where the Jacobian matrix might become singular and because of poor initializations $[7,8,19]$. Therefore, fixed-point iteration methods are robust and popular techniques for Richards equation even if they converge linearly. Let $m$ be the number of the nonlinear iteration and $J$ the Jacobian matrix computed from (11). Both Newton-Raphson and fixed-point iteration schemes read:

$$
\left\{\begin{array}{l}
J\left(h^{n+1, m}\right) \delta h=-R\left(h^{n+1, m}\right), \\
h^{n+1, m+1}=h^{n+1, m}+\delta h .
\end{array}\right.
$$

In this study, the fixed-point iteration method is chosen and constructed like in [7] by avoiding the first derivative terms inside the Jacobian matrix. The stopping criterion is $\|R(h)\|_{L^{2}(\Omega)}<\varepsilon$ where $\varepsilon$ is a user-defined tolerance.

Time adaptation is motivated by the convergence of the nonlinear solver. Indeed, solution takes too many iterations or even fails to converge by oscillating because of stiff initialization or severe boundary conditions. On one hand, transient simulations have difficulties to converge if the time step is too large but, on the other hand, shorter time steps mean more time steps and so, a longer computational time. That is the reason why time adaptation is very attractive and common for Richards equation. However, it exists different strategies to adjust the time step [1]: first ones are heuristic and mainly based on convergence performance of the nonlinear solver; second ones are rational and based on error control. The latter are generally much more efficient than the former. Yet, heuristic methods may outperform them in regards of their simple implementation.

For this study, the time step is adjusted heuristically according to the previous number of iterations $N_{i t}$ from the nonlinear solver such as [9, 20]. The simulations begins with a time step $\delta t^{0}$. The next time step $\delta t^{n+1}$ is calculated from the previous one $\delta t^{n}$ according to the time-stepping scheme:

$$
\left\{\begin{array}{l}
\delta t^{n+1}= \begin{cases}\lambda_{\mathrm{amp}} \delta t^{n} & \text { if } N_{i t} \leq \text { inf It } \\
\delta t^{n} & \text { if } i n f I t<N_{i t} \leq \text { supIt } \\
\lambda_{\text {red }} \delta t^{n} & \text { if } \operatorname{supIt}<N_{i t} \leq \max I t\end{cases} \\
\delta t^{n}=\lambda_{\text {red }} \delta t^{n} \text { if } \max I t<N_{i t} \text { or if the solver has failed (time step is started again). }
\end{array}\right.
$$

The factors $0<\lambda_{\text {red }}<1$ and $1<\lambda_{\text {amp }}$ as well as the threshold values $0<$ inf It $\leq$ supIt $<$ maxIt are prescribed by the user. A minimum time step is defined to avoid excessive small time step. With this approach, the nonlinear solver is more robust. Indeed, whatever $\delta t^{0}$, the time step is adjusted until the nonlinear solver succeeds to converge. Then, thanks to the coefficients, the time step is adjusted smoothly. Nevertheless, the method depends on empirical parameters and does not provide an optimal time step because the amplification/reduction coefficients restrict the possible size of the new time step. It is difficult to assess if computational time is saved for the simulation in regards of convergence due to balance between nonlinear iterations and time iterations.

\subsection{Adaptive mesh refinement}

Solution of Richards equation holds sharp wetting fronts which are moving dynamically through the domain among smooth regions [11]. They arise typically from boundary conditions and dissipate over time by coming across the saturated zone. Mesh adaptation is a technique which can handle these particularities to reach the desired accuracy. Recent strategies involve both size and order adaptation, based on a posteriori errors [10] or interpolation error [18]. In this study, an $h$ adaptation based on a simple a posteriori error is performed like Miller et al. [11]. Yet, whereas they use changes in effective saturation, that's directly changes in the solution, the gradient of hydraulic head $h$, which serves as error indicator. The latter has the advantage of working both for the saturated and unsaturated regions.

Mesh adaptation is a 2D block-based technique adapted from Altazin et al. [21]. The process of 
mesh adaptation begins with the evaluation of the criterion defined by:

$$
C_{E}=\frac{1}{\max _{E \in \mathcal{E}_{h}}\left(\frac{1}{|E|}\|\nabla h\|_{L^{2}(E)}\right)} \frac{1}{|E|}\|\nabla h\|_{L^{2}(E)} .
$$

The criterion is normalized so that $0 \leq C_{E} \leq 1$. The second step consists in deciding to refine, coarsen or do nothing thanks to user-defined threshold values $0<\beta_{\min } \leq \beta_{\max }<1$ for the criterion. Elements are merged or split according to the scheme described in Figure 1. A quadtree graph-based numbering is used to define easily neighbouring elements of faces. To avoid excessive refinement and coarsening, minimum and maximum levels are set up. Then, the mesh is smoothed by checking that each neighbouring block of elements cannot have a difference of refinement level greater than one.
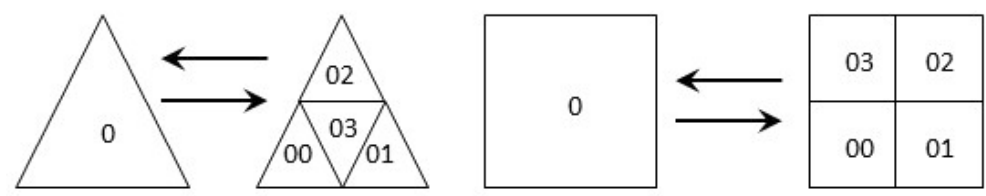

Figure 1: Refinement and coarsening process for one triangular and quadrangular element

In the last step, the solution is projected from the previous mesh to the new one by solving a local discontinuous Galerkin problem. The element-wise weak formulation corresponding to $h_{E_{\text {new }}}=h_{E_{\text {old }}}$ reads:

$$
\int_{E_{\text {new }}} h_{E_{\text {new }}} v \mathrm{~d} x=\int_{E_{\text {new }}} h_{E_{\text {old }}} v \mathrm{~d} x .
$$

This strategy of adaptation is simple but the a posteriori indicator requires empiric parameters to balance computational performance and accuracy. This forces to calibrate $\beta_{\min }$ ans $\beta_{\max }$ for each simulation. These limits are described in [22] as well as a procedure to overcome them.

\section{Numerical results}

\subsection{One-dimensional infiltration numerical test case}

This numerical test-case was firstly introduced by Celia et al. [23] on the basis of an experiment from Haverkamp et al. [15]. It was taken afterwards by others [24] and corresponds to a class of classical test cases, for example, see [11]. This problem deals with an 1D-infiltration into a soil column of $40 \mathrm{~cm}$ height and $8 \mathrm{~cm}$ width. $\mathbb{K}$ and $\theta$ are taken from (2) with $A=1.175 \times 10^{6}$, $B=4.74, C=1.611 \times 10^{6}, D=3.96, K_{s}=0.00944 \mathrm{~cm} / \mathrm{s}, \theta_{s}=0.287$ and $\theta_{r}=0.075$. The initial condition is $h_{0}=-61.5+z \mathrm{~cm}$. On the top and bottom, Dirichlet boundary conditions for hydraulic head are prescribed: $h_{t o p}=19.3 \mathrm{~cm}, h_{\text {bottom }}=-61.5 \mathrm{~cm}$ which results in downward infiltration. Sides are impervious. Simulation parameters are $p=1, \sigma_{i}=\sigma_{b}=100$ and $T=600 \mathrm{~s}$. Threshold values for adaptive mesh refinement are $: \beta_{\min }=\beta_{\max }=0.5$.

The solutions are in very good agreement to one another as seen for pressure head in the Figure 2a. Moreover, $h$ adaptation is capturing the wetting front efficiently as it is shown in Figure $2 \mathrm{~b}$.

\subsection{Comparison with water table drainage experiment}

Vauclin et al. made a drainage experiment of a slab of soil in which experimental details and positions of water table can be found [16]. A water table depletion occurs after an instantaneous drop of the imposed hydraulic head. The box is $200 \mathrm{~cm} \times 300 \mathrm{~cm}$. $\mathbb{K}$ and $\theta$ are taken from (2) with $A=359720.49, B=4.5, C=40000, D=2.9, K_{s}=40 \mathrm{~cm} / h, \theta_{s}=0.3$ and $\theta_{r}=0$. This experiment is used as a test-case to show a transient simulation with seepage boundary condition. The domain is initially in hydrostatic equilibrium: $h_{0}=145 \mathrm{~cm}$. Boundary conditions at the bottom, at the top, on the right and on the left for $z>145 \mathrm{~cm}$ are $\nabla h \cdot \boldsymbol{n}=0$. On the left, the 
level is kept constant in the ditch for $z \leq 75 \mathrm{~cm}, h=75 \mathrm{~cm}$, and, the seepage boundary condition is prescribed for $45 \mathrm{~cm}<z \leq 145 \mathrm{~cm}$.

Numerical simulation is performed with $p=1, \sigma_{i}=\sigma_{b}=100$ on a mesh with $\Delta x=10 \mathrm{~cm}$ and $\Delta z=5 \mathrm{~cm}$. Water table location matches $\psi=0$. Numerical positions of water table are displayed in Figure 3 together with the experimental data from [16].

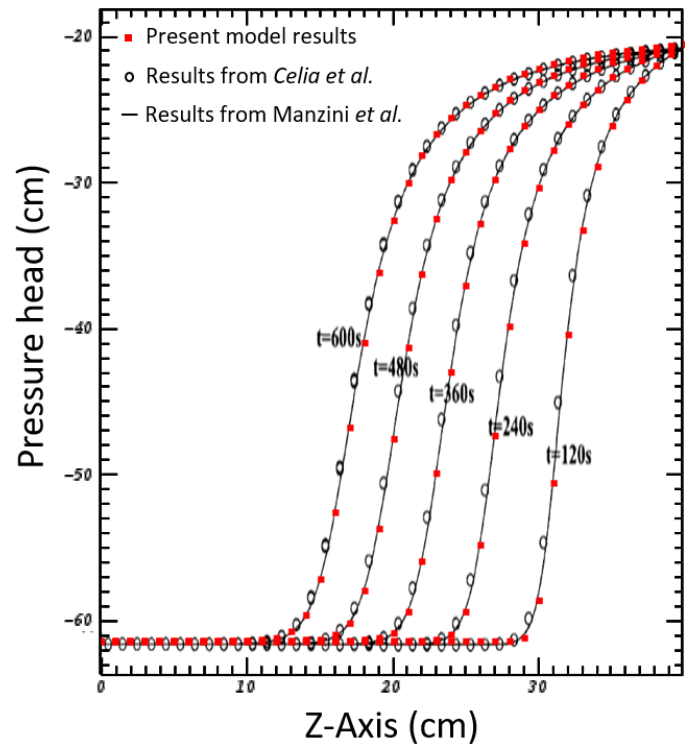

(a) Pressure head profiles adapted from Manzini et al. [24]

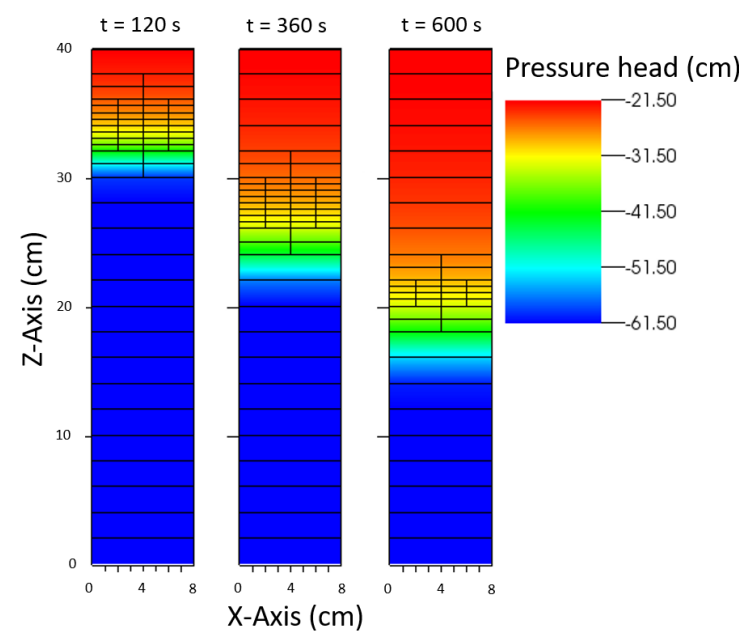

(b) Adaptive mesh refinement around the wetting front

Figure 2: Results for the one-dimensional infiltration numerical test case

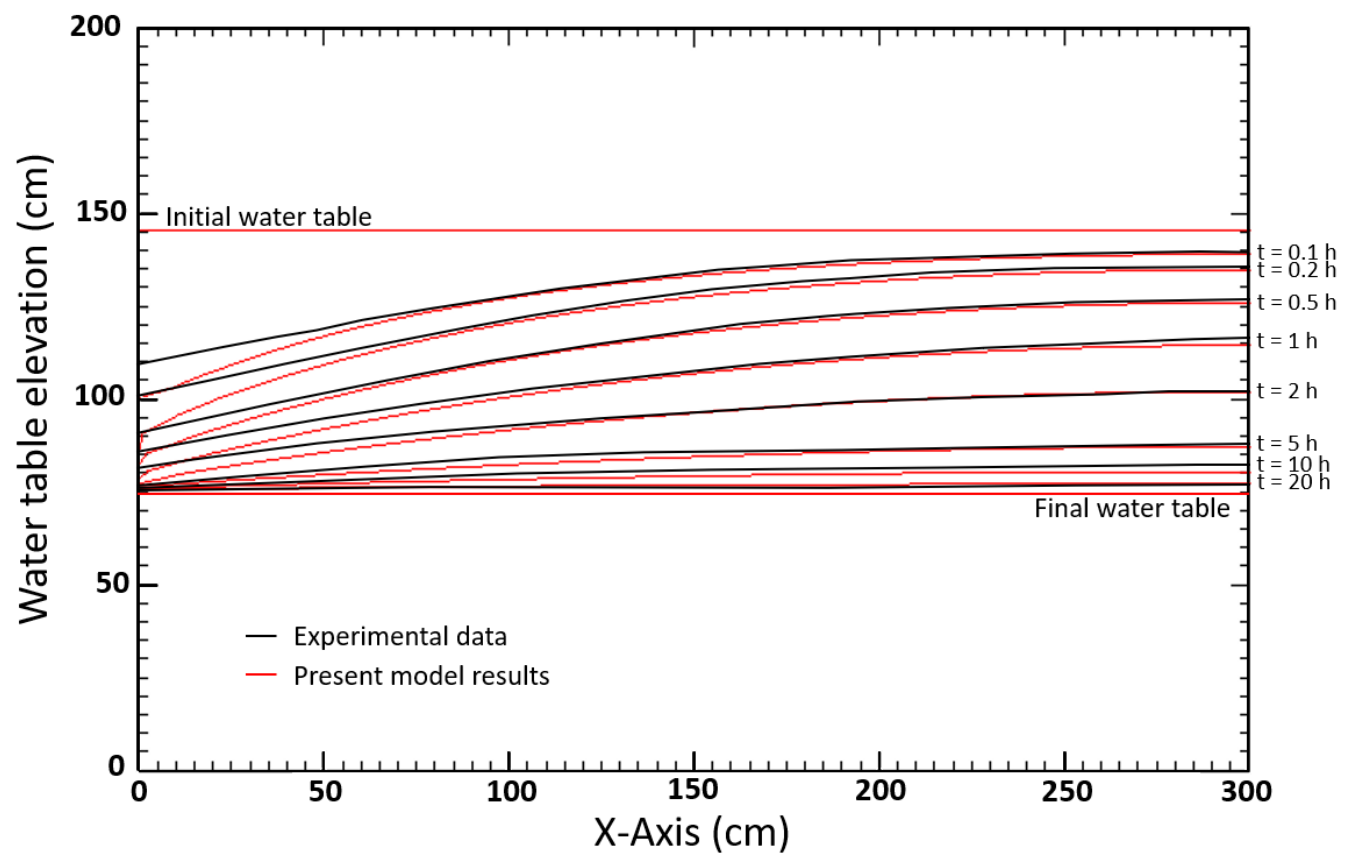

Figure 3: Numerical simulation of two-dimensional drainage with water table position compared to experimental data from Vauclin et al. [16]

Results presented in the Figure 3 show that the model is able to reproduce satisfactory the transient water table dynamics. However, disagreements near the seepage boundary are found be- 
tween numerical and experimental profiles. Two reasons can explain it. One is in relation with the experimental set-up because water should not pour out completely freely through the experimental device in such a way that seepage boundary condition does not model it properly. Another reason is the determination of hydraulic properties which was done by correlation from measurements with some fairly important dispersion, due to natural heterogeneity and by neglecting hysteresis[16]. This would explain some discrepancies. In every instance, it is not linked to discretization because results does not change whatever the mesh and order approximation are chosen. Other numerical studies based on this experiment are reported [9, 16, 25]. They also struggle to match water table profiles near the seepage.

\section{Conclusion}

In this paper, Richards equation is discretized: a discontinuous Galerkin method associated with a backward Euler scheme is able to simulate variably saturated flows in porous media for a wide range of problems, including seepage boundary condition. Richards equation gives rise to some issues concerning non-linearities and convergence behaviour. In order to provide a computationally simple but efficient algorithm in terms of solution accuracy and robustness, the nonlinear solver is based on a fixed-point iteration method with adaptive time stepping. Besides, an adaptive mesh refinement technique is employed to deal with steep gradients. Finally, simulations illustrate how the method performs for numerical and experimental test-cases.

Further research is underway to extend adaptive mesh refinement to an efficient $h p$-adaptation, assess more carefully the convergence behaviour of seepage boundary condition and make time step adaptation less heuristic.

\section{Acknowledgment}

This work has been supported financially by Provence-Alpes-Côte d'Azur region (France).

\section{References}

[1] Farthing, M. W. \& Ogden, F. L.: Numerical Solution of Richards' Equation: A Review of Advances and Challenges. Soil Science Society of America Journal, vol. 81, no. 6: (2017) pp. 1257-1269.

[2] Zha, Y., Yang, J., Zeng, J., Tso, C.-H. M., Zeng, W. \& Shi, L.: Review of numerical solution of Richardson-Richards equation for variably saturated flow in soils. Wiley Interdisciplinary Reviews: Water, vol. 6, no. 5: (2019).

[3] Clément, J.-B., Ersoy, M., Golay, F. \& Sous, D.: Discontinuous Galerkin method for steadystate Richards equation. In Topical Problems of Fluid Mechanics, Institute of Thermomechanics, AS CR, v.v.i., D. Šimurda and T. Bodnár, Ed., Prague: (2019) pp. 53-62.

[4] Rubin, J.: Theoretical analysis of two-dimensional, transient flow of water in unsaturated and partly unsaturated soils. Soil Science Society of America Journal, vol. 32, no. 5: (1968) pp. 607-615.

[5] Cooley, R. L.: A finite difference method for unsteady flow in variably saturated porous media: Application to a single pumping well. Water Resources Research, vol. 7, no. 6: (1971) pp. 16071625.

[6] Wilhelm Alt, H. \& Luckhaus, S.: Quasilinear elliptic-parabolic differential equations. Mathematische Zeitschrift, vol. 183, no. 3: (1983) pp. 311-341.

[7] Lehmann, F. \& Ackerer, P.: Comparison of iterative methods for improved solutions of the fluid flow equation in partially saturated porous media. Transport in Porous Media, vol. 31, no. 3: (1998) pp. 275-292. 
[8] Paniconi, C. \& Putti, M.: A comparison of Picard and Newton iteration in the numerical solution of multidimensional variably saturated flow problems. Water Resources Research, vol. 30, no. 12: (1994) pp. 3357-3374.

[9] Thoms, R. B., Johnson, R. L. \& Healy, R. W.: User's guide to the variably saturated flow (VSF) process to MODFLOW. U.S. Geological Survey, Techniques and Methods 6-A18: (2006).

[10] Li, H., Farthing, M. \& Miller, C.: Adaptive local discontinuous Galerkin approximation to Richards' equation. Advances in Water Resources, vol. 30, no. 9: (2007) pp. 1883-1901.

[11] Miller, C. T., Abhishek, C. \& Farthing, M. W.: A spatially and temporally adaptive solution of Richards' equation. Advances in Water Resources, vol. 29, no. 4: (2006) pp. 525-545.

[12] Rivière, B.: Discontinuous Galerkin Methods for Solving Elliptic and Parabolic Equations. Society for Industrial and Applied Mathematics: (2008).

[13] Dolejší, V. \& Feistauer, M.: Discontinuous Galerkin Method. Springer International Publishing: (2015).

[14] Szymkiewicz, A.: Modelling Water Flow in Unsaturated Porous Media. Springer Berlin Heidelberg: (2013).

[15] Haverkamp, R., Vauclin, M., Touma, J., Wierenga, P. J. \& Vachaud, G.: A comparison of numerical simulation models for one-dimensional infiltration. Soil Science Society of America Journal, vol. 41, no. 2: (1977) pp. 285-294.

[16] Vauclin, M., Vachaud, G. \& Khanji, J.: Two dimensional numerical analysis of transient water transfer in saturated-unsaturated soils. In Computer Simulation of Water Resources Systems, IFIP Work. Conf., G. Vansteenkiste, Ed., Amsterdam, North-holland Publishing company: (1975) pp. 299-323.

[17] Scudeler, C., Paniconi, C., Pasetto, D. \& Putti, M.: Examination of the seepage face boundary condition in subsurface and coupled surface/subsurface hydrological models. Water Resources Research, vol. 53, no. 3: (2017) pp. 1799-1819.

[18] Dolejší, V., Kuraz, M. \& Solin, P.: Adaptive higher-order space-time discontinuous Galerkin method for the computer simulation of variably-saturated porous media flows. Applied Mathematical Modelling, vol. 72: (2019) pp. 276-305.

[19] List, F. \& Radu, F. A.: A study on iterative methods for solving Richards' equation. Computational Geosciences, vol. 20, no. 2: (2016) pp. 341-353.

[20] Bergamaschi, L. \& Putti, M.: Mixed finite elements and newton-type linearizations for the solution of Richards' equation. International Journal for Numerical Methods in Engineering, vol. 45, no. 8: (1999) pp. 1025-1046.

[21] Altazin, T., Ersoy, M., Golay, F., Sous, D. \& Yushchenko, L.: Numerical investigation of BB-AMR scheme using entropy production as refinement criterion. International Journal of Computational Fluid Dynamics, vol. 30, no. 3: (2016) pp. 256-271.

[22] Pons, K., and Ersoy, M.: Adaptive mesh refinement method. Part 1: Automatic thresholding based on a distribution function. Working paper or preprint, https://hal.archivesouvertes.fr/hal-01330679v2, submitted: (2019).

[23] Celia, M. A., Bouloutas, E. T. \& Zarba, R. L.: A general mass-conservative numerical solution for the unsaturated flow equation. Water Resources Research, vol. 26, no. 7: (1990) pp. 14831496.

[24] Manzini, G. \& Ferraris, S.: Mass-conservative finite volume methods on 2-d unstructured grids for the Richards' equation. Advances in Water Resources, vol. 27, no. 12: (2004) pp. 1199-1215.

[25] Clement, T., Wise, W. R. \& Molz, F. J.: A physically based, two-dimensional, finite-difference algorithm for modeling variably saturated flow. Journal of Hydrology, vol. 161, no. 1-4: (1994) pp. $71-90$. 